\title{
Discussion on Education and Teaching of Natural Ecological Color Design Based on Virtual Environment Construction
}

\author{
Min Xie, Min Xie *, Sisi Shang \\ The School of Arts, Hubei University of Education, Wuhan, Hubei, China \\ *Corresponding Author.
}

\begin{abstract}
:
As one of the elements of natural ecological display design, color is particularly important for the virtual construction of natural ecology. However, how to reflect the ecological nature in the natural ecological display color design is a problem worthy of thinking. This paper focuses on the ecological nature of display color design. Taking the ecological nature of color design of Beijing Natural Ecological Exhibition Hall as an example, it starts from the definition of ecological nature of display color design to the embodiment of ecological nature of color design. Then this paper focuses on the ecological expression of display color design with the theme of nature. Finally, this paper discusses the teaching method of natural ecological color design education, and puts forward the teaching method guided by the virtual construction process of natural ecology and the natural color design method. Experimental results show that the method can improve the effectiveness of color design education.
\end{abstract}

Keywords: Color, Natural Ecology, Exhibition Design, Art Education and Teaching Methods.

\section{INTRODUCTION}

In recent years, the application of virtual reality is increasingly widespread, and has brought huge economic and social benefits. Virtual reality (VR) comes from the English "virtual reality". It was first proposed by J. Lanier in 1989, and also translated into "spiritual realm" and "unreal reality" [1-2]. It is a kind of "natural human-computer interface" rising in the 1990s. Published in the International Journal of virtual reality in 1995, the glossary of virtual reality terms is defined as follows: virtual reality is a kind of computer system that creates an artificial world [3]. By using virtual reality technology, people can simulate the feeling of being in the scene and be able to manipulate the objects in it.

At the world electronics annual meeting in 1993, American scientists g. burdea and ecoiffet put forward the theory of "triangle of virtual reality" in the paper "virtual reality systems and Applications", which concisely expressed the basic characteristics of virtual reality, namely: three "I" (immersion, interaction, imagination) [4-5]. At present, virtual reality can be divided into desktop, immersive, enhanced and distributed according to the degree of interaction and immersion and the scope of users. From the above discussion, we can infer that virtual reality is essentially an advanced and intelligent computer user interface, which provides users with visual, auditory, tactile and other intuitive and natural perceptual interaction modes 
in real time.

\section{CHARACTERISTICS OF VIRTUAL REALITY ART DESIGN}

\subsection{Features of works}

The most prominent feature of the product in the digital age is its immateriality. Because the composition of digital art and design works is not arranged by molecules or atoms. It's the arrangement of bits and bytes [6]. According to Negroponte, a professor at the Massachusetts Institute of technology, bits have no color, size and weight, but they can "virtually" represent all kinds of our world [7-8]. Therefore, the virtual "world" with bits as the smallest element is called the immaterial world, in order to distinguish the real world.

Virtual reality is digital and virtual in form, but it should reflect the information of the real world. Users need to get a sense of reality when browsing it, which determines that there must be a corresponding relationship between it and the real world, that is, all kinds of attributes and characteristics of the virtual reality world must simulate and visualize the real scenes and natural phenomena in the real world according to the physical logic and objective laws of the real world [9]. The inextricably linked with reality, namely reality, is also the difference between it and other art forms.

From the above non-material characteristics, we know that virtual reality art design works are separated from the "material" attribute of traditional art design works and are no longer limited by material attributes [10]. Digital technology means that everything is programmable, and programmable means that there is no dream that can not be realized absolutely. Therefore, the virtual world originates from the real world, and can also transcend the real world. Compared with the real world and traditional art design works, the transcendence of the virtual reality art design works makes it unique charm.

Virtual reality is the intersection and compatibility of time and space art. It is its integration characteristics that make it compatible with and invade all the art design fields in the past, learn from all the traditional art design means of expression, and complete its own art design creation. It is also its integrated feature, which is compatible with visual, auditory, even tactile, olfactory and taste elements, which leads to the user blurring the difference between reality and virtual, as if they are in the real world, so as to realize the experience of the real world.

\subsection{Creative features}

Like traditional art design, virtual reality art design is a purposeful and planned activity. Different from art, the way of creation is guided by needs and based on technology.

Artistic design creation can be roughly divided into three stages: (1) capture and excavate people's needs, analyze the corresponding forms according to the needs, and initially determine the appearance characteristics and practical methods considering the current technology, technology, cost, painting and sculpture and other artistic styles. (2) The engineer examines the technical possibility of the conception of the work, transits from the technical concept to the structure and form of the work, and corrects the form considering the technical feasibility; (3) the art designer and the engineer jointly reprocess the work technically and aesthetically.

Virtual reality modeling is a complex process, which needs to express the visual, auditory, tactile information of the observer quantitatively or qualitatively in a certain way. The so-called quantification is to obtain the perceptual data by using the relevant technology, and thus describe the virtual world accurately; the so-called qualitative is a representative global graph summarized from a large amount of information. 
This large amount of information may not be represented by perceptual data, but some useful conclusions can be concluded from it.

It is an important step to make virtual reality by using software tools. This process mainly completes two tasks: drawing computer graphics and defining application interface. Therefore, the virtual reality production method (as shown in Figure 1) is made by the virtual reality production engine, supported by the virtual reality software toolkit. The virtual reality system is made to interact with users through interface devices (I / O devices) and virtual reality devices, thus realizing a virtual world.

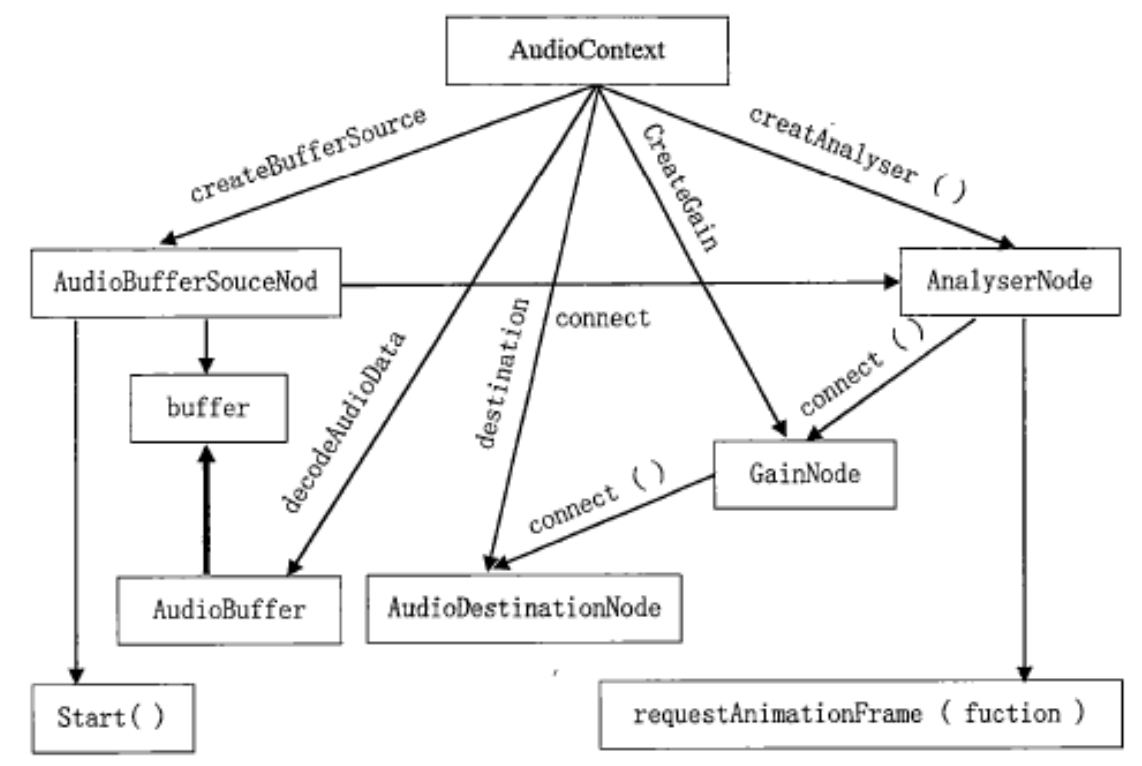

Fig 1: The making method of virtual reality

Virtual reality works have both the characteristics of "reality" and "transcendence". Among them, reality shows that the virtual world originates from the real world, and the attributes of virtual objects should be selected from the attributes of real objects. Transcendence also determines that the selection process requires designers to give full play to their imagination and creativity. Therefore, the creative process of virtual reality art design is a creative choice process. The creation process of virtual reality needs to choose part of the attributes of the real world to reflect the real world, so that people can get the real experience and feelings to meet the needs of various industries, which is also the fundamental significance of virtual simulation. Virtual reality must grasp the logic and physical rules of the real world, and choose the attributes of the real world according to people's needs.

\section{HUMAN FACTORS INFLUENCING THE ART DESIGN OF VIRTUAL REALITY}

3.1 The way people perceive the world establishes the elements of virtual reality art design

The most important source of human visual perception is $80 \%$. Vision is the most complex and important sense of human beings, and it is also the most comprehensive and extensive way of perception.

Hearing is an important feeling inferior to vision. 10-15\% of the information in the external world is perceived by human beings through hearing. Hearing can enhance people's realistic experience of the environment on the basis of vision. For example, when we watch a sound movie, we will be more impressed than when we watch a silent movie. Therefore, how to improve the quality of sound and the degree of harmony between sound and image is one of the art design elements of virtual reality. 
Vision and hearing are the necessary perception channels of virtual reality, whether for desktop virtual reality, immersive virtual reality, augmented virtual reality or distributed virtual reality. Different from the visual and auditory effects provided by traditional art forms, virtual reality provides dynamic and all-round real-time rendering of three-dimensional audio-visual effects, which makes it easier for people to feel personally.

Touch, smell and taste account for about $5 \%$ of the information people get from the outside world.

People perceive the virtual world through vision, hearing, touch, smell and taste. So, if we study how to make virtual reality serve people better from the aspect of object, we should also start with the visual elements, auditory elements, tactile elements, smell and taste elements.

\subsection{The overall effect of human needs analysis on Virtual Reality Design}

Bruce Asher put forward a systematic method for designers in the British design magazine No. 172-181, which reflects the procedures of determining the goals in the process of general art design. This paper only selects the first half of this procedure, that is, the procedure of functional orientation.

(1) Preparation. It includes input of question content, evaluation of question, evaluation of expected design work and preparation of temporary answers;

(2) Simple synthesis. It includes inputting indicative requirements, setting goals and limiting specific conditions;

(3) Put forward the project. It includes summing up all kinds of temporary problems and putting forward suggestions on ways to take;

(4) Collect all kinds of data. It includes centralizing all data and classifying and storing data;

(5) Analysis. It includes decomposing the problem into several sub items, analyzing the sub items according to the objectives, preparing to put forward the functional indicators, and re evaluating the project and its cost.

It can be seen that the function orientation is based on the comprehensive consideration of customer needs, technical conditions and production costs. The specific methods of function orientation in virtual reality art design are as follows:

First of all, determine the basic function, what is the use, determine whether it is the simulation of reality or the creation of reality, in what field, entertainment, military, education or product design, etc.

Secondly, according to the basic functions, the user group and other functions needed by the user group are located. Different user groups have different aesthetic tastes and world views. For example, the entertainment customer group is mainly young people, and the theme and game scene can be specially designed according to the age and cultural background of the main customer group; the education and training customer group is mainly school, and on the basis of completing the education function, the students' interest can be highlighted.

Thirdly, according to the basic function and the characteristics of user group, the aesthetic function is positioned. The rational application of formal beauty laws such as sense of order, master and subordinate, and diversity and unification can also help to improve the user satisfaction of virtual environment. Aesthetic function should be consistent with its use, and the user's demand determines the position of "beauty" in the works.

Finally, according to the above three aspects of the function positioning, it is determined to use desktop virtual reality system, immersion virtual reality system or distributed virtual reality system; determine the specific production means, whether to use three-dimensional model, two-dimensional pictures or 
combination of the two. Different types and means require different equipment, which requires appropriate solutions based on existing technology and cost.

\section{VISUAL ELEMENTS ANALYSIS OF VIRTUAL REALITY ART DESIGN}

\subsection{The realization steps of visual elements}

The representation of virtual object's color, material and texture is to assign the original object's color, material and other attributes to the geometric model, and then give the material changes in the environment by lighting. This makes the visual elements of virtual reality art design have their own characteristics

The first step is to establish the geometric description of the virtual 3D scene with mathematical method. How to describe the scene is very important, it directly affects the complexity of the model and the way of texture mapping, as well as the amount of machine time, we must choose a reasonable and effective means of data representation and input.

In the second step, because the two-dimensional views are displayed on the computer screen (or the large projection screen, or the display screen of the helmet mounted display), it is necessary to convert the threedimensional geometric description into the two-dimensional view, which is completed by changing the perspective of the scene.

Third, in the actual field of view, not all objects in the scene and all faces of objects can be seen. For example, some faces are blocked by other objects. Therefore, it is necessary to use some algorithm (such as hidden surface elimination algorithm) to erase the invisible surface outside the field of view or covered by other objects.

The fourth step, in order to achieve realistic visual effect, we should also according to the observer's position, distance and angle, light and color all the objects in the scene, so that they have a real texture. From the point of view of the document on computer software, it is equivalent to a process of "Compiling" the various attributes (or parameters) of the frame diagram, such as what texture to use, what color to apply, and which direction to shine, etc. It can even express such attributes as whether there is elasticity, whether it can penetrate, how much weight, etc.

The fifth step, through hardware devices (such as helmet mounted display, 3D glasses, etc.) stereo display.

4.2 Modeling features of virtual objects

(1) Realism

The realism of modeling comes from the reality of virtual reality works. Virtual reality art design is different from traditional art design forms, such as lamp design. The space for designers to imagine is very large. As long as the lighting function is satisfied, the shape of lamps can have various forms with great differences. Virtual objects are not the same, except in the field of entertainment, modeling is mostly the description of real objects, like realistic art. The difference is that this kind of description is not for the purpose of "aesthetic feeling", but for the purpose of "application".

(2) Adapt to dynamic real time display

Traditional image display does not require real-time. Traditional painting is a static, solidified form of space; later appeared animation, how long each picture takes to generate is not limited. But the dynamic display of virtual reality is realized in the process of interaction with users. The image should change with the position of the observer's eyes, and it should be able to be generated quickly to give people a sense of reality. So not only static frame state but also dynamic real-time display should be considered in modeling. 
In addition to the perfection of volume, structure, proportion and other factors, we should also make it adapt to dynamic interaction.

(3) The solid is impenetrable

This is derived from the previous feature. In order to ensure that users can truly see the dynamic performance of virtual objects, solid objects in the virtual environment need to be impenetrable. Impenetrability is realized by collision detection technology. It is collision detection that can avoid the occurrence of unreal dynamic situations such as people passing through the wall.

4.3 The abstraction of Art

The art abstract image will be more concise, generalized and powerful than the natural image. The art abstract method can simplify the model without affecting the simulation effect. Using this technique, for example, to create a bird model in creator (Figure 2), we only need to abstract the trend of key parts (including wings, abdomen, etc.) and grasp the outline of the body.
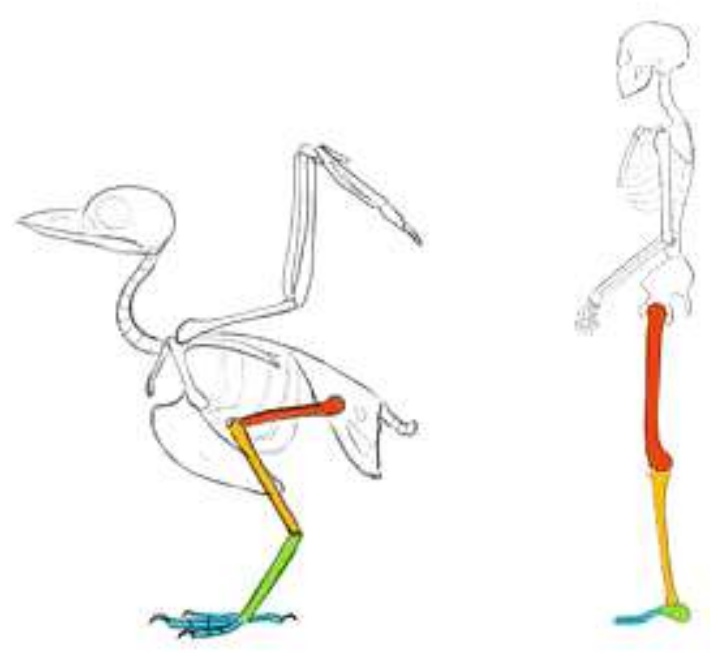

Fig 2: A model of a bird

In "three dimensional plastic arts" published by China Radio University Press, such a "shape formula" has been mentioned: the number of points + relative distance and position $=$ shape feeling. The point in the formula is the shape support point we imagine. The number, distance and position distribution of the points play an important role in the shape. This formula is an important tool for us to understand morphology and analyze 2D and 3D morphology (as shown in Figure 3).
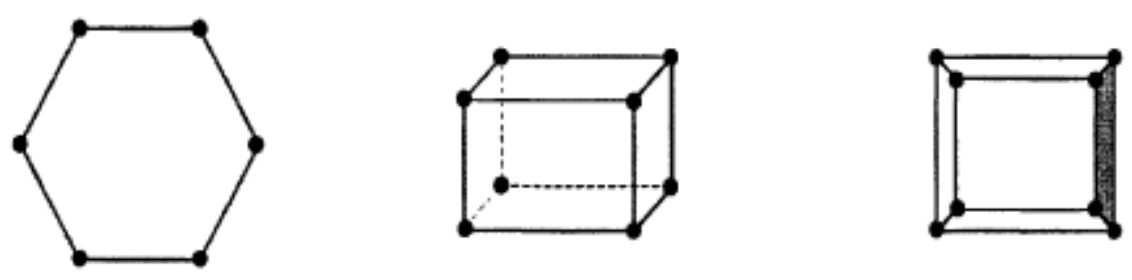

Fig 3: Basic form and supporting point

The idea of virtual 3D modeling in model making is very similar to that of traditional 3D modeling, and its geometric shape is also determined by the number, distance and distribution of key points. In creator, the construction process of geometric model (Figure 4) is essentially the process of establishing geometric shape 
by the position and number of key points, that is, the supporting points of shape are determined by observing real objects, and then the outline is refined and enriched.

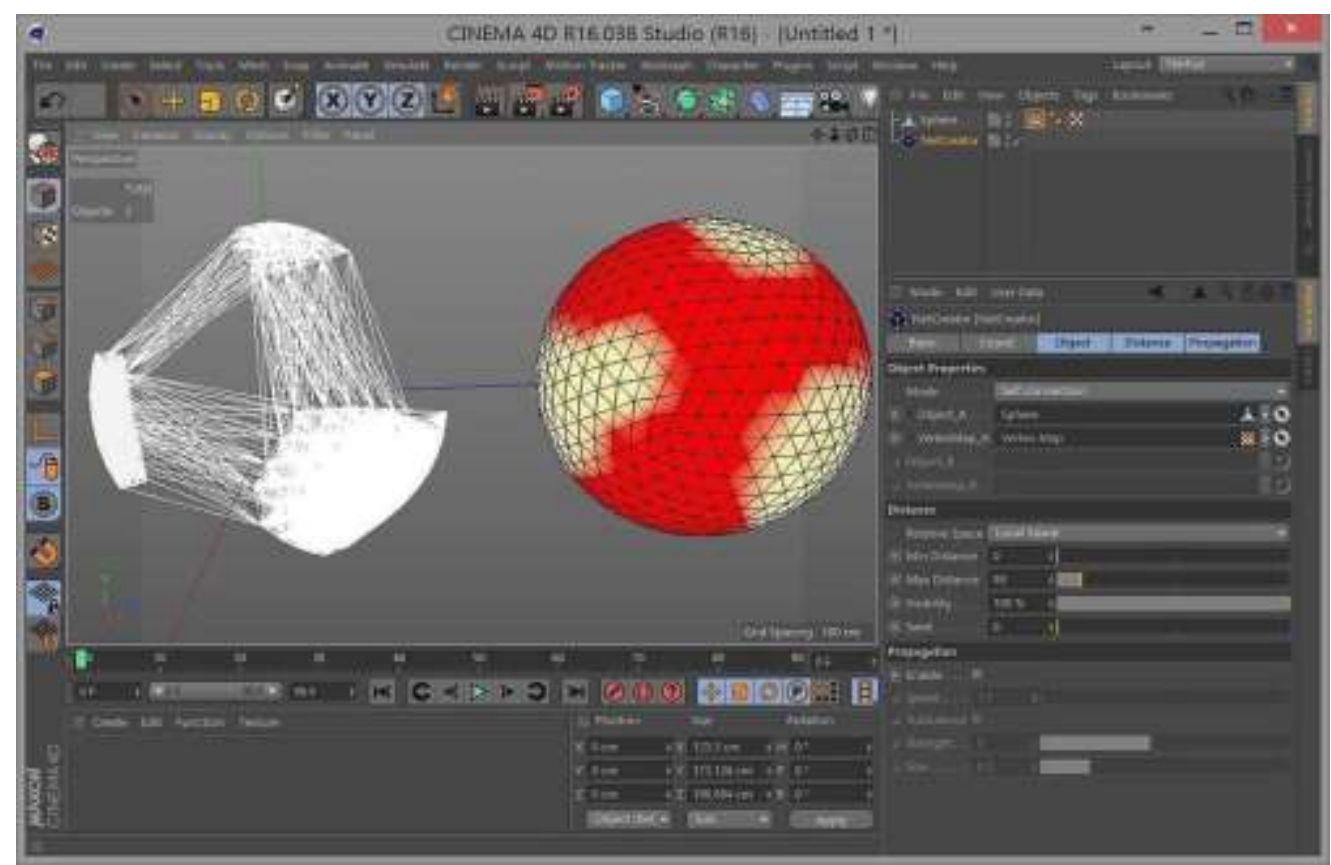

Fig 4: Creator modeling

\section{CONCLUSION}

Nowadays, virtual reality is widely used. How to improve the simulation effect has become a new research hotspot. This paper attempts to use the idea of art design to improve the simulation effect of virtual reality, that is, to deal with the relationship between art and technology based on human needs. Improving the simulation effect depends on the improvement of the matching degree between user needs and virtual world attributes.

\section{REFERENCES}

[1] Li Chuan. Experimental Study on the Evaluation Model of College English Online Teaching. Foreign Language and Foreign Language Teaching, 2005 (07): 33-36

[2] Zhong Binglin. Analysis on Some Hot Issues of Undergraduate Teaching Evaluation -- Also on the System Design and Implementation Framework of the New Round of Evaluation. Higher Education Research, 2009 (06): 38-45

[3] Zhang Linlin. Examining the Management of University Teaching Archives from the Perspective of Teaching Evaluation. Office Business, 2017, 07 (no.264): 125-125

[4] Li Haixia, Zhang Zhifeng, Wu Yan. Effect Evaluation of Micro Video Assisted Physiology Theory Teaching Based on Functional Experiment. Chinese Journal of Medical Education Exploration, 2020, Vol. 19, No. 10, 1168-1171

[5] Li Ying, Song Haoming, Yang Jun. Application of Simulated Case and Group Discussion Teaching Method in Clinical Thinking Training Course. Chinese Journal of Medical Education, 2020, 40 (12): 983-987 
[6] Zhang Zhiying, Zhang Yantong. Analysis and Reflection on Undergraduate Teaching Evaluation. China Management Science, 2005, 13 (1): 136-140

[7] Dai Weidong, Zhang Xuemei. On Teaching Evaluation and Discipline Construction of English Majors. China Foreign Languages, 2005 (02): 6-9

[8] Zhong Binglin. Let Undergraduate Teaching Evaluation Be Improved in the Process of Re Understanding and Re Thinking. China Higher Education, 2006, 000 (007): 4-7

[9] Du Zhimin. Establishing a Long-term Mechanism of Internal Teaching Evaluation to Ensure the Improvement of Teaching Quality. China Higher Education, 2007, 000 (010): 48-50

[10] Li Yadong, Wang Sunyu. the Publication of Teaching Evaluation Results from Two Different Evaluations. Higher Education Research, 2002, 023 (001): 79-82 